\title{
La calcolosi renale in un laboratorio di patologia clinica: modello organizzativo e nuove tecniche analitiche
}

Giornale di Tecniche Nefrologiche e Dialitiche 2019, Vol. 3I(I) 22-29

(C) The Author(s) 2019

Article reuse guidelines:

sagepub.com/journals-permissions

DOI: $10.1177 / 0394936219836635$

journals.sagepub.com/home/gtn

(3)SAGE

\author{
Giovanni Cangiano', Grazia Buccino', Sara La Palombara', \\ Marianna Bencivenga ${ }^{2}$, Roberta Annecchini², \\ Giovanna Capolongo ${ }^{3}$, Mariano Conticelli ${ }^{4}$, \\ Marco Terribile ${ }^{5}$ e Antonio Sarappa'
}

\begin{abstract}
Nephrolithiasis: how the laboratory of clinical pathology can play a pivotal role in the prevention of lithiasic events

We here describe our 15-year experience and report both on methodological problems (proposing a new datasheet and biochemical techniques) and on organizational ones (we discuss our patient approach and useful reporting for the physician).
\end{abstract}

\section{Keywords}

Nephrolithiasis, Metabolic study, Organizational model, Laboratory techniques

\section{Introduzione}

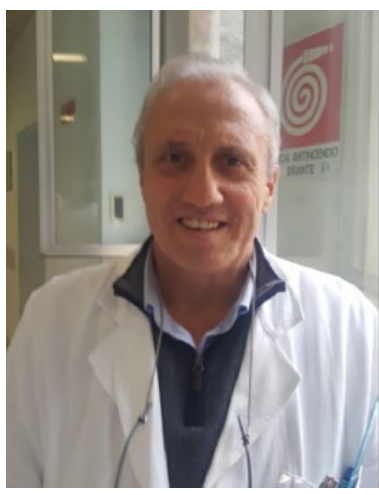

L'apporto del Laboratorio di Patologia Clinica allo studio dei pazienti affetti da calcolosi renale può essere utile a prevenire gli eventi litiasici. Un accurato screening metabolico effettuato sulle urine delle 24 ore può evidenziare la saturazione di alcuni analiti e, pertanto, la tendenza alla formazione di calcolirenali.

Il nostro Laboratorio, attivo dall'anno 2002 per lo studio delle calcolosi, ha dovuto nel tempo affrontare nuove problematiche analitiche e strumentali di "nicchia", cambiando quindi radicalmente l'organizzazione e l'approccio al paziente affetto da calcolosi renale.

Nel seguente lavoro vengono esposte le modalità con cui il laboratorista gestisce i rapporti con il paziente ed il nefrologo richiedente. Si propongono inoltre nuovi dosaggi biochimici e procedure informatiche, costruiti in Laboratorio, ed adattati allo studio della calcolosi renale.

\section{Organizzazione di lavoro e metodologia}

I. Fase pre-analitica: preparazione allo studio metabolico

Il paziente che afferisce al nostro Laboratorio, indirizzato dal medico di medicina generale o dallo specialista, richiede analiti urinari da dosare sulla raccolta delle 24 ore e sulle urine estemporanee.

Il laboratorista delle calcolosi raccoglie i dati anagrafici e tutte le altre notizie utili alle determinazioni (es. assunzione di farmaci). Viene inoltre rilasciato agli

'Laboratorio di Patologia Clinica - P.O. dei Pellegrini ASL NA I Centro, Napoli, Italy

${ }^{2}$ Nefrologia e Dialisi - P.O. dei Pellegrini ASL NA I Centro, Napoli, Italy

${ }^{3}$ Nefrologia e Dialisi - Ospedale del Mare ASL NA I Centro, Napoli,

Italy

${ }^{4}$ Laboratorio di Patologia Clinica - Ospedale del Mare ASL NA I

Centro, Napoli, Italy

5Urologia - Università della Campania Luigi Vanvitelli, Napoli, Italy

Corrispondenza:

Giovanni Cangiano, Laboratorio di Patologia Clinica - P.O. dei Pellegrini ASL NA I Centro, Via Comunale del Principe I3/a, Napoli, Italy.

E-mail: gianni.cangiano@libero.it 


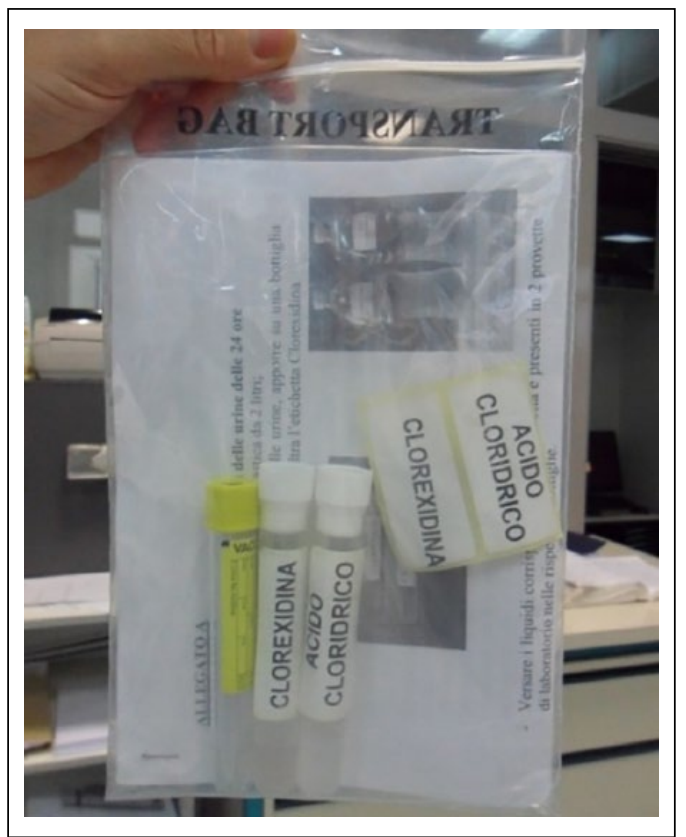

Figura I. Kit per i casi di calcolosi.

interessati un apposito kit (figura 1) contenente un foglio d'istruzioni per la raccolta urinaria, etichette adesive per $\mathrm{i}$ contenitori, modulistica per eventuali richieste a carico del Servizio Sanitario Nazionale, due provette contenenti conservanti pericolosi (una con $5 \mathrm{~mL}$ di acido cloridrico $37 \%$ e l'altra con $2 \mathrm{~mL}$ di clorexidina digluconato $20 \%$ ) ed infine il relativo modulo di assunzione di responsabilità da firmare. Il team di laboratoristi (generalmente operanti in Biochimica Clinica) illustra nel dettaglio la modalità di prelievo (come da istruzioni presenti nel kit), ne spiega anche il motivo di tale raccolta "sdoppiata", avverte il paziente sulla pericolosità dei liquidi da usare (norme precauzionali sono indicate nelle istruzioni) ed eventualmente rimanda il paziente ad un link scientifico di laboratorio, menzionato sempre nel foglio di istruzioni, in cui si parla di calcolosi in generale ma principalmente delle specifiche tecniche di laboratorio utilizzate.

Il paziente effettua la raccolta scartando l'urina al risveglio e raccogliendo tutte le minzioni fino alla prima urina del mattino seguente. Per ogni minzione, il paziente divide ogni volta l'urina in due aliquote che vengono poi riposte in due differenti recipienti da 2.5 litri (figura 2). Il modulo da compilare per l'assunzione di responsabilità all'uso delle sostanze pericolose e per la ricezione del referto per posta elettronica è illustrato in figura 3 .

La prima provetta contiene $5 \mathrm{~mL}$ di acido cloridrico al $37 \%$, necessario ad evitare la formazione dei precipitati di calcio. ${ }^{1}$ Nella seconda provetta sono invece presenti $2 \mathrm{~mL}$ di clorexidina, necessaria alla determinazione di alcuni analiti urinari ma principalmente utile per la determinazione del $\mathrm{pH}$ e dell'acido urico. La clorexidina infatti è un potente battericida che impedisce la degradazione dell'urea

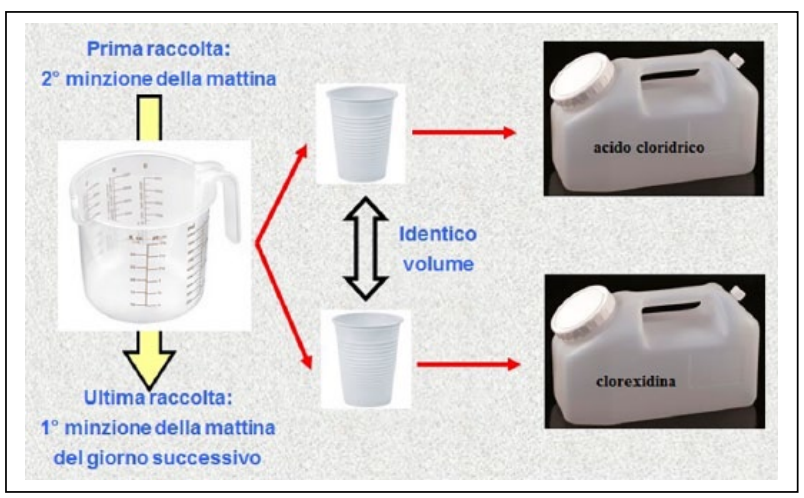

Figura 2. Modalità di raccolta delle urine delle 24 ore.

e quindi l'alcalinizzazione delle urine. Ad ogni minzione è fondamentale la miscelazione dei liquidi contenuti nelle provette con le urine. La diuresi totale delle 24 ore è rappresentata dalla somma dei volumi raccolti nei due contenitori. ${ }^{2}$ Viene inoltre richiesta una raccolta di urine della mattina (urine a digiuno: minzione successiva a quella delle 24 ore e raccolta prima di colazione). ${ }^{3} \mathrm{~A}$ fine raccolta, il paziente consegna al Laboratorio i due contenitori delle 24 ore (contenenti urina preservata con acido cloridrico e clorexidina) e la provetta con urina della mattina; vengono inoltre consegnate anche le provette vuote che contenevano i conservanti.

Nelle urine della mattina si misurano creatinina e calcio, mentre in quelle della giornata, si misurano calcio, ossalato, magnesio, citrato, solfato, fosforo, sodio (su urine acidificate), potassio, cloro, ammonio, acido urico, creatinina, proteine, urea e $\mathrm{pH}$ (su urine in clorexidina) (figura 4). ${ }^{4}$

La preparazione dei campioni per l'ossalato si effettua diluendo $1 \mathrm{~mL}$ di urina con $1 \mathrm{~mL}$ di tampone EDTA a pH 7.0. Si controlla il relativo $\mathrm{pH}$ ed eventualmente lo si corregge con $\mathrm{HCl} 1 \mathrm{~N}$ o con $\mathrm{NaOH} 1 \mathrm{~N}$ fino ad ottenere un valore compreso tra 5.0 e 7.0. Si versa quindi questa miscela in un tubo di purificazione contenente carbone attivo. Si agita per 5 minuti tramite vortex e poi si centrifuga. Il sovranatante raccolto viene conservato in frigo e successivamente testato su strumentazione analitica.

\section{Fase pre-analitica: dissoluzione del calcolo renale pervenuto in laboratorio}

Circa $30 \mathrm{mg}$ di calcolo frantumato (2 misurini rasi da 15 $\mathrm{mg}$ ) si solubilizzano con 3 gocce di acido solforico concentrato. Successivamente si aggiungono $10 \mathrm{~mL}$ di acqua distillata. Si miscela accuratamente e poi si centrifuga. Il sopranatante è pronto per i dosaggi di acido urico, ammonio, calcio, fosfato e cistina, tranne che per quello dell'ossalato: la determinazione di quest'ultimo necessita infatti di una ulteriore diluizione 1:3 con tampone EDTA a pH 7.0.5 


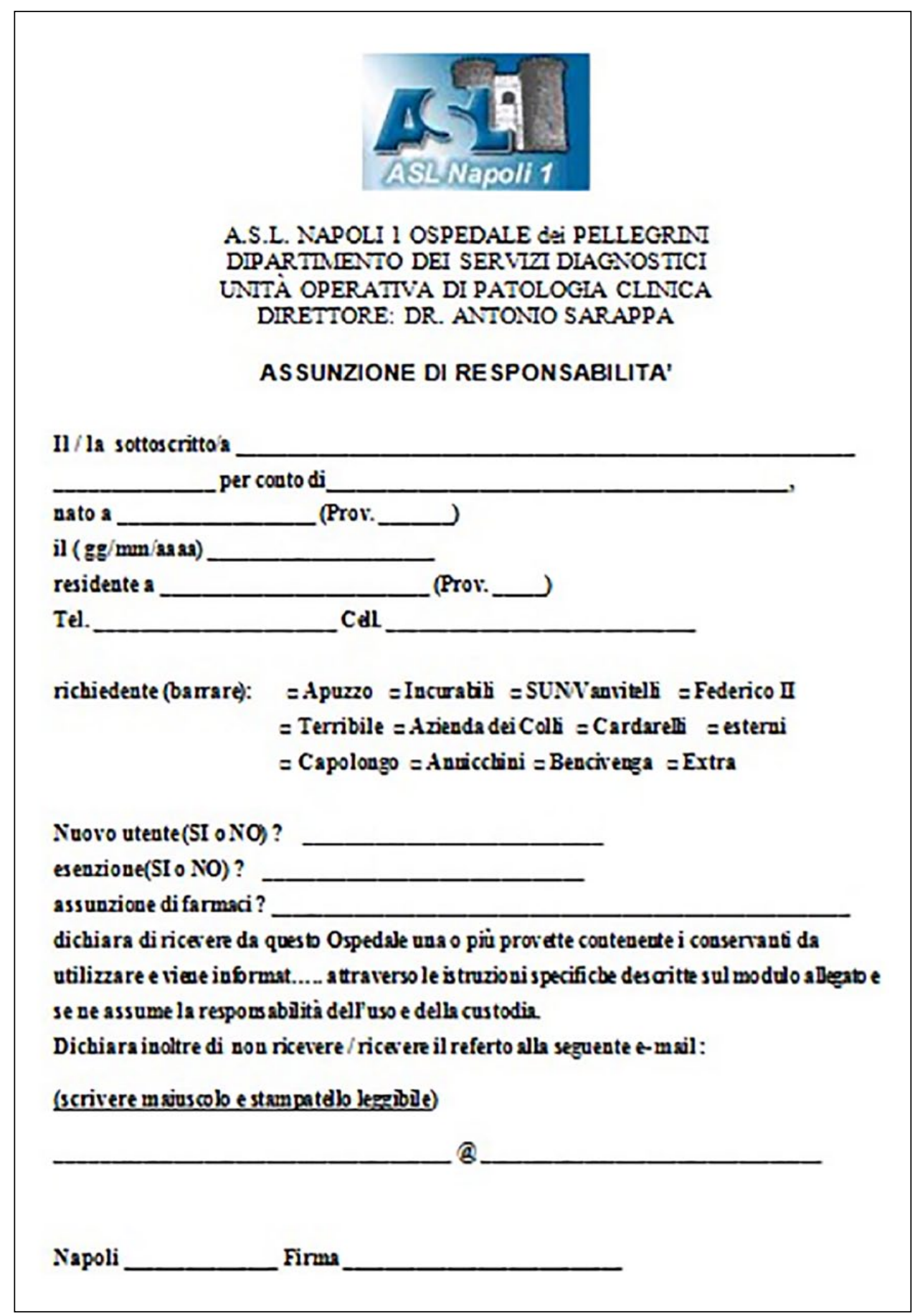

Figura 3. Modulo per l'assunzione di responsabilità.

\section{Metodologie analitiche}

Le determinazioni urinarie di calcio, fosforo, magnesio, sodio, potassio, cloro, acido urico, creatinina, proteine, urea ed ammonio (per l'ammonio si effettua una diluizione 1:100 con acqua distillata) si effettuano su analizzatore biochimico di routine, nel nostro caso il COBAS 6000 (Roche Diagnostics).
Il dosaggio dei citrati, ossalati, cistina e solfati, è effettuato settimanalmente su strumentazione biochimica Viva E (Siemens Helthyneers).

Nel nostro laboratorio si usano i reagenti della ditta BSN (Biological Sales Network) per le determinazioni dei citrati: si usa il metodo UV con lettura del NADH a $340 \mathrm{~nm}^{6,7}$ e dell'ossalato, col metodo Trinder, misurato colorimetricamente a $600 \mathrm{~nm} .{ }^{8,9} \mathrm{Il}$ dosaggio degli ossalati 
è utilizzato anche per calcolarne la concentrazione nel calcolo renale.

Le determinazioni del solfato e della cistina si effettuano con metodi fotometrici quantitativi da noi validati in laboratorio. La determinazione del solfato si basa sulla reazione con cloruro di bario per formazione di solfato di bario, misurato turbidimetricamente alla lunghezza d'onda di $436 \mathrm{~nm}$ : la concentrazione finale si calcola tramite curva di calibrazione a 6 punti (da 0 a $400 \mathrm{mg} / \mathrm{dL}){ }^{9-11}$

La determinazione fotometrica della cistina si basa sulla reazione con acido fosfotungstico: in ambiente tamponato a $\mathrm{pH} 5.5$, per aggiunta di $220 \mu \mathrm{L}$ di una soluzione costituita da tampone acetato $2 \mathrm{M}$ ed acqua (in rapporto 5:3.5), la cistina viene ridotta a cisteina per aggiunta di $30 \mu \mathrm{L}$ di solfito di sodio $0.1 \mathrm{M}$ che, in presenza di $120 \mu \mathrm{L}$ di acido fosfotungstico $30 \%$ forma il blu di tungsteno, la cui concentrazione viene misurata a $660 \mathrm{~nm}$. Possibili interferenti quali acido urico, acido ascorbico e altri disolfuri vengono eliminati grazie ad un "bianco campione" in cui nel primo reagente è presente tampone acetato e cloruro mercurico $0.1 \mathrm{M}$ : la concentrazione della cistina si calcola sottraendo la concentrazione del bianco a quella del campione calcolata mediante curva di calibrazione a 6 punti da 0 a $40 \mathrm{mg} / \mathrm{dL}$. Lo stesso dosaggio è utilizzato anche per calcolare la concentrazione di cistina nel calcolo renale. ${ }^{12-17}$

\section{Fase post-analitica: refertazione dello studio metabolico}

Nella refertazione dello studio metabolico i parametri urinari vengono riferiti a valori soglia nelle 24 ore basati su un ampio consenso della letteratura ${ }^{18}$ e non ad intervalli di riferimento.

Grazie al software in Access da noi sviluppato ${ }^{19}$ è possibile refertare tutti gli analiti urinari misurati nelle 24 ore e nelle urine del mattino, nonché registrare le relative supersaturazioni di calcio ossalato (SSR CaOx), calcio fosfato monoacido (SSR Ca HPO4), acido urico (SSR UA) e cistina (SSR Cys), estrapolate in precedenza con il software Litho Risk. ${ }^{18}$ I valori patologici vengono evidenziati in grassetto e viene inoltre riportato un istogramma in cui sono raggruppati i parametri metabolici, ambientali e le supersaturazioni. Le colonne superiori dell'istogramma, colorate in rosso, rappresentano i valori patologici. In questo specifico referto è presente inoltre un commento finale (figura 5).

\section{Fase post-analitica: refertazione del calcolo urinario}

Come per lo studio metabolico, anche per la determinazione dei 7 parametri biochimici più facilmente riscontrabili in un comune calcolo urinariosi effettuano i dosaggi

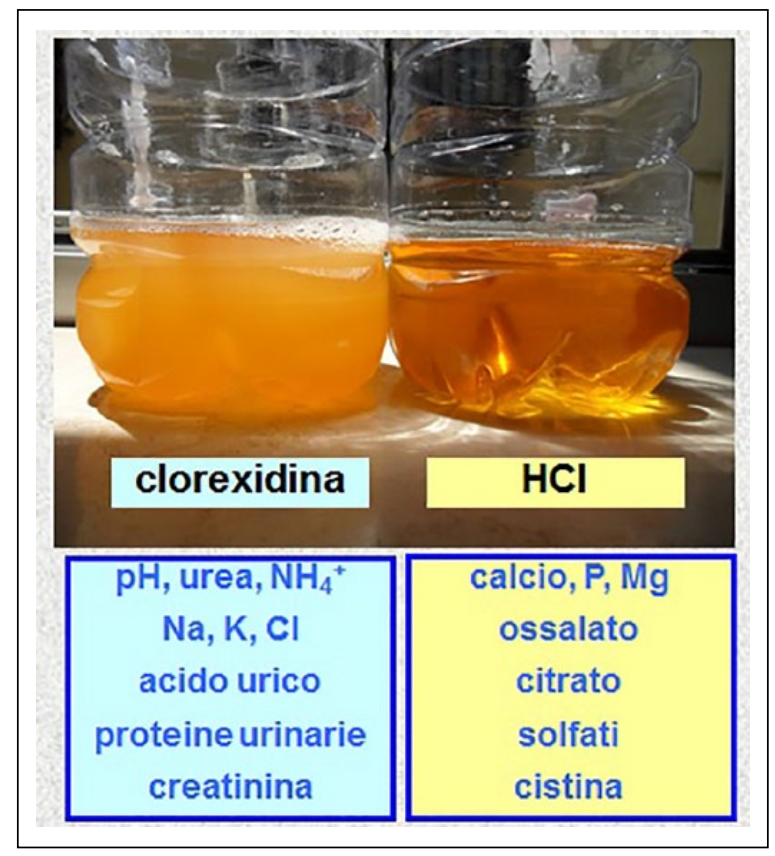

Figura 4. Analiti urinari determinati sulla raccolta doppia delle $24 \mathrm{~h}$.

biochimici su Cobas 6000 (calcio, fosforo, acido urico, magnesio ed ammonio) e su Viva E (ossalati e cistina).

Dopo opportuna preparazione della soluzione, così come descritto in precedenza, le diverse concentrazioni ottenute (calcio, fosforo, magnesio, acido urico e cistinain $\mathrm{mg} / \mathrm{dL}$, ossalato in $\mathrm{mmol} / \mathrm{L}$ ed ammonio in $\mu \mathrm{g} / \mathrm{dL}$ ) si inseriscono in un nostro programma sviluppato in Excel. 5,20 Tale programma provvede alla conversione di tutte le concentrazioni in $\mathrm{mg} / \mathrm{dL}$ ed alla relativa percentualizzazione delle 7 componenti chimiche. Coni pesi molecolari si ricavano inoltre le relative concentrazioni in $\mu \mathrm{mol} / \mathrm{dL}$.

Le micromoli/dL di struvite si estrapolano dal confronto delle micromoli/dL di fosforo, magnesio ed ammonio: la più piccola concentrazione rilevata di una di queste tre componenti costituisce anche la concentrazione di struvite.

$\mathrm{Si}$ confrontano poi le micromoli/dL di calcio ed ossalato: la concentrazione inferiore rappresenta anche il contenuto di calcio ossalato.

Le micromoli/dL di calcio e di fosforo in eccesso vengono tra loro rapportate: a seconda del valore del rapporto calcio/fosforo ottenuto, si ricava il tipo di "calcio fosfato" (brushite: $\mathrm{Ca} / \mathrm{P}=1.00$; fosfato octacalcico: $\mathrm{Ca} / \mathrm{P}=1.33$; whitlockite: $\mathrm{Ca} / \mathrm{P}=1.50$; idrossiapatite: $\mathrm{Ca} / \mathrm{P}=1.67$; apatite: $\mathrm{Ca} / \mathrm{P}=2.00$ ).

Una quota di acido urico può combinarsi con le rispettive micromoli/dL di ammonio (in rapporto equimolecolare) per evidenziare la concentrazione, sempre in $\mu \mathrm{mol} / \mathrm{dL}$, di urato di ammonio.

Dal peso molecolare dei vari sali ottenuti, si ricavano i mg/dL. Ciò vale anche per i rimanenti contributi delle 
singole componenti chimiche (residuo inorganico). Sarà quindi facile ottenere la percentualizzazione di tutti i sali e del residuo. La refertazione del nostro calcolo si effettua grazie al programma in Access usato anche per la gestione dello studio metabolico.

\section{Discussione}

Un'adeguata formazione del personale di Chimica Clinica del nostro Laboratorio e l'uso di semplici kit per la raccolta urinaria, preparati periodicamente dagli stessi operatori, facilita notevolmente l'approccio al paziente affetto da calcolosi renale senza gravare minimamente sul lavoro routinario e d'urgenza.

Per le determinazioni di solfato, citrato, ossalato, ma principalmente per la cistina (metodica da noi implementata) si preferisce l'uso dell'analizzatore da banco Viva E Siemens, normalmente usato per il dosaggio degli immunosoppressori. Mentre i dosaggi urinari di routine si effettuano su Cobas 6000, o comunque su altra strumentazione biochimica.

La strumentazione Viva E è infatti pratica, rapida e facilmente programmabile anche per un esame complesso come quello per la determinazione della cistina che prevede una doppia misurazione (campione totale e bianco) e l'uso di tre reagenti, stabili nel tempo. La metodica "bianco" per la cistina si diversifica da quella "totale" per avere nel primo reagente la presenza di cloruro mercurico: pertanto, dopo aver ottenuto la concentrazione di cistina "totale", si effettua semplicemente la sostituzione del primo reagente e si ripete il dosaggio dei campioni. Per differenza tra la prima e la seconda determinazione (bianco) si ottiene pertanto il valore finale della concentrazione di cistina.

L'analizzatore biochimico Viva E appare inoltre di grande utilità nella rilevazione dei solfati (metodica turbidimetrica da noi costruita), nonché in quella di ossalati e citrati, ambedue metodiche commercializzate per dosaggi manuali e da noi facilmente implementate sullo strumento.

Le sedute di lavoro, per quanto riguarda le determinazioni effettuate su Viva E, hanno cadenza settimanale, mentre gli altri dosaggi, eseguiti su Cobas 6000 , si praticano entro le 24 ore dall'arrivo del campione così come la misurazione del volume urinario, la rilevazione potenziometrica del $\mathrm{pH}$ e la purificazione del campione per la determinazione dell'ossalato. Un'intera giornata di lavoro (ore 8-20) è sufficiente al completamento di circa dieci studi metabolici che necessitano di un unico operatore, se laureato, per la validazione ed il commento finale. La quantificazione del costo dei reagenti (15 dosaggi, compresa la cistinuria e determinazione potenziometrica del $\mathrm{pH}$ ) non supera i dieci euro. L'estrapolazione delle supersaturazioni renali richiede un tempo di circa trenta minuti.
Lo studio del rischio litogeno si gestisce abbastanza facilmente grazie all'uso di un software in Access da noi creato. La stampa dell'esame comprende un unico foglio di facile lettura in cui si compattano tutti gli analiti delle 24 ore, il rapporto calcio-creatinina eseguito su urine estemporanee, le supersaturazioni urinarie relative (SSR) di calcio ossalato, calcio fosfato, acido urico, cistina e degli istogrammi relativi ai parametri metabolici, ambientali ed alle SSR. Un commento finale, esclusivamente laboratoristico, può sicuramente dare ulteriori indicazioni al clinico e/o al nefrologo per gli opportuni provvedimenti alimentari e/o farmacologici da intraprendere. Il referto finale, trasformato in formato pdf, viene poi trasmesso per posta elettronica sia al paziente che al medico richiedente (figura 5).

Il rapporto cistina/creatinina urinaria è molto utilizzato in ambiente pediatrico: campioni diluiti e negativi per presenza di cistinuria (con dosaggi inferiori a $150 \mathrm{mg} / 24$ ore) possono invece essere indicativi di eventuale difetto tubulare se il sopraccitato rapporto è superiore a $0.050 .{ }^{14}$ L'eventuale positività per cistina, evidenziata col nostro test all'acido fosfotungstico, dovrà essere confermata con tecniche cromatografiche.

La determinazione qualitativa e quantitativa delle diverse componenti contenute in un calcolo urinario è poco specifica se effettuata con i tradizionali kit in commercio, specie se riferita alla presenza di più sali associati a "calcio fosfato". La procedura da noi proposta effettua i più importanti dosaggi (acido urico, ossalato, ammonio, calcio, fosforo, cistina e magnesio) in fotometria e, grazie ad uno specifico programma in Excel da noi creato, riesce a mettere in evidenza anche le diverse tipologie di calcio fosfato con la relativa percentualizzazione. La stampa finale con l'immagine del calcolo (figura 6) viene gestita col programma in Access già citato e usato per lo studio metabolico.

La differenziazione tra whewellite e weddellite, nonché la caratterizzazione di calcoli rari (xantina e diidrossiadenina), si effettua con la tecnica all'infrarosso (la cui strumentazione ha un costo di circa 15,000 euro). Rispetto al kit tradizionale dei calcoli eseguiti, in uso nella stragrande maggioranza dei laboratori, la nostra proposta fotometrica ha lo stesso costo quantificato intorno ai 10 euro; invece la metodica di riferimento all'infrarosso, se richiesta in regime privato, viene proposta ad un costo raddoppiato.

La metodica fotometrica all'acido fosfotungstico da noi proposta per la determinazione di screening della cistinuria è sufficiente per il controllo terapeutico dei pazienti afferenti al nostro Presidio Ospedaliero ed alle Aziende Pediatriche viciniori. Tale dosaggio è affidabile, ha un costo bassissimo (meno di un euro) e necessita di un tempo di circa 30 minuti (calibrazione e controlli compresi) e sostituisce il vecchio test di Brand, poco 


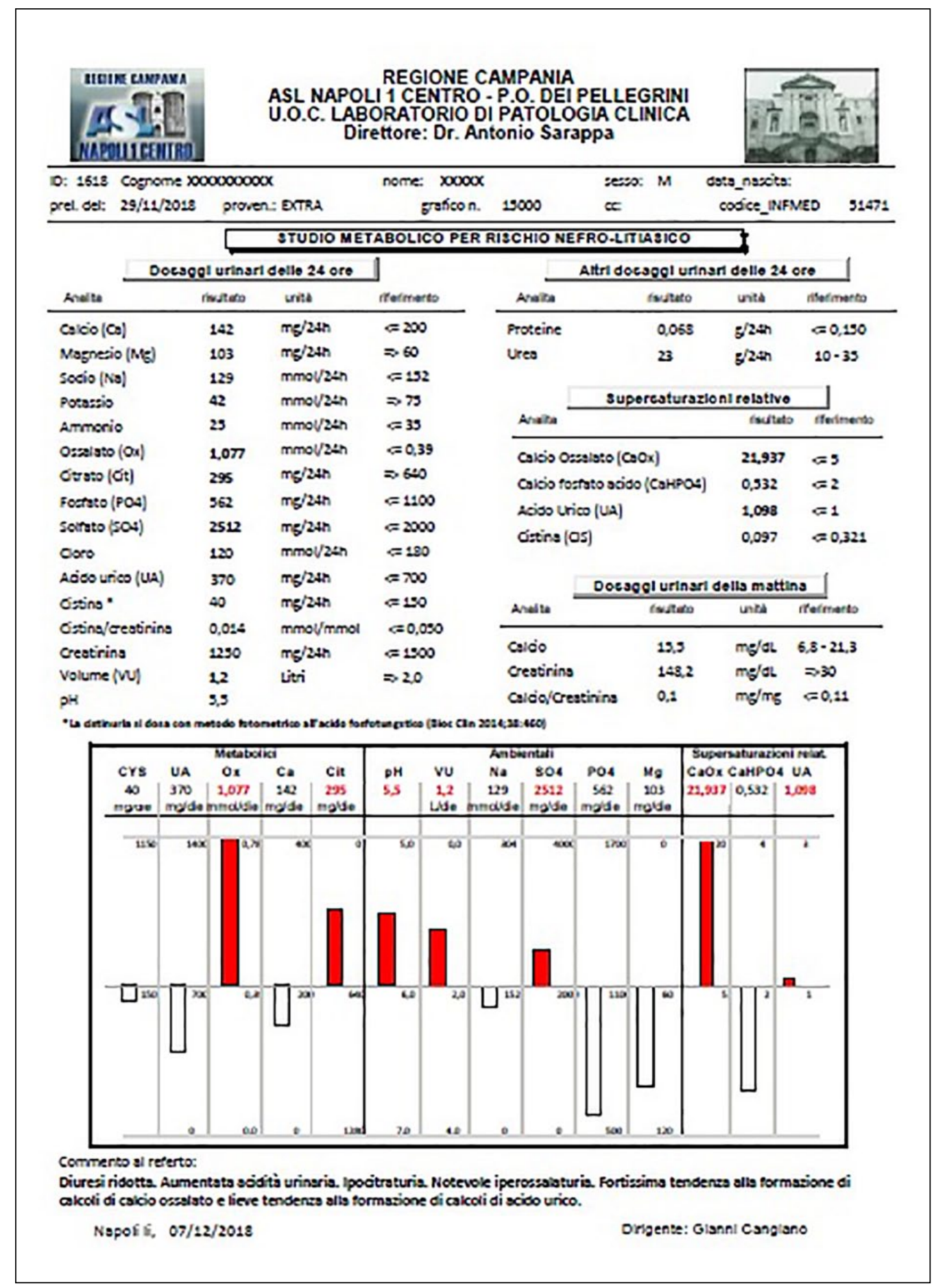

Figura 5. Referto dello studio metabolico.

specifico e non utilizzabile in laboratorio per la presenza di cianuro.

Per quanto riguarda le ormai sempre più numerose richieste di determinazione dello studio metabolico, appare evidente che le motivazioni siano da attribuire sicuramente alla sua utilità nel prevenire le recidive litiasiche (controlli dei pazienti una o due volte all'anno) ma anche al costo abbastanza contenuto (circa una cinquantina di euro) e a tempi di consegna sufficientemente ragionevoli (circa una settimana). 


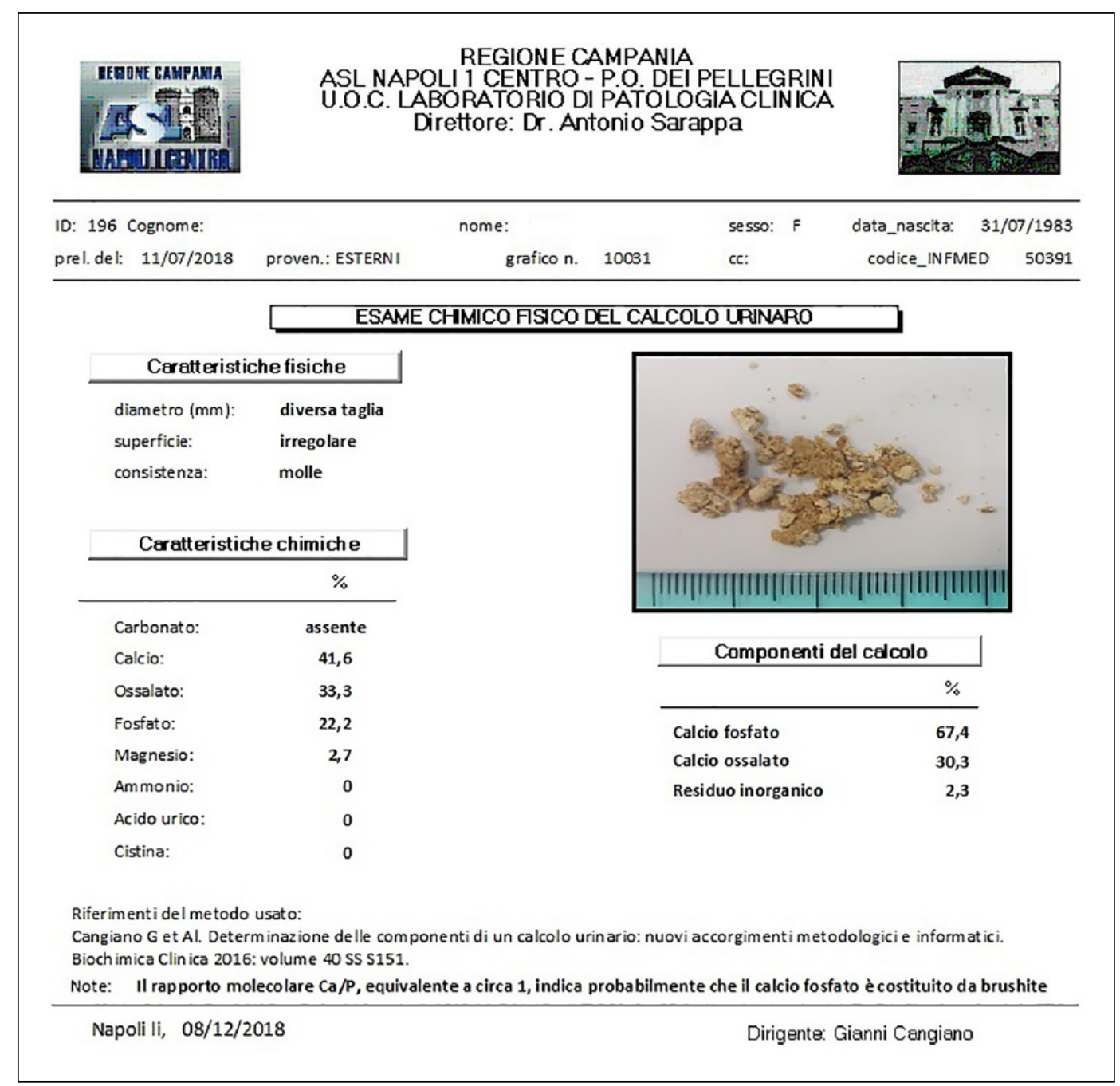

Figura 6. Referto del calcolo urinario.

\section{Dichiarazione di assenza di conflitto di interessi}

Gli Autori dichiarano di non avere conflitti di interessi.

\section{Finanziamenti}

Gli Autori dichiarano di non aver ricevuto finanziamenti specifici da qualsiasi ente nei settori pubblico, privato o senza fini di lucro.

\section{Bibliografia}

1. Bandinelli R, Pasquinelli F. Calcio, Fosfati, Magnesio. Diagnostica e tecniche di laboratorio. Chimica Clinica. Ed. Rosini, Firenze 1979; 26:1067.

2. Di Maina E, Forte F, Risitano A, et al. Diagnosi di laboratorio nello screening metabolico dei pazienti nefrolitiasici: nostra esperienza. Il patologo clinico 2003; 9-10: 234-8.

3. Caudarella R. Ipercalciuria idiopatica. L'endocrinologo 2007; 8:209-20.
4. Cangiano G, Russo M, Forte F, et al. Diagnostica di laboratorio della calcolosi renale. Biologi Italiani 2008; 8:41.

5. Cangiano G, Buccino G, Errico A, et al. Determinazione delle componenti di un calcolo urinario: nuovi accorgimenti metodologici ed informatici. Biochimica clinica 2016; 40SS:S151.

6. Latte A, Cangiano G, Russo M, et al. Determinazione dei citrati urinari su AU 400 Olympus. Biochimica Clinica 2009; 33:488.

7. Cangiano G, Russo M, Forte F, et al. Determinazione fotometriche automatizzate di citrato ed ossalato urinario. Biologi Italiani 2010; 5/6:21-25.

8. Cangiano G, Latte A, Russo M, et al. Dosaggio degli ossalati urinari su AU 400 Olympus. Biochimica Clinica 2010; 34:536.

9. Cangiano G, Latte A, Di Maina E, et al. Determinazione dei solfati e degli ossalati urinari: ulteriori accorgimenti metodologici. Biochimica Clinica 2012; 36:498.

10. Latte A, Cangiano G, Russo M, et al. Dosaggio fotometrico dei solfati urinari. Biochimica Clinica 2006; 30:398. 
11. Latte A, Cangiano G, Russo M, et al. Dosaggio dei solfati urinari su AU600 Olympus Biochimica Clinica 2007; 31:509.

12. Latte A, Cangiano G, Russo M, et al. Screening della cistinuria (metodo al fosfotungstato) su AU600 Olympus e proposta di intervalli di riferimento. Biochimica Clinica 2005; 29:268.

13. Cangiano $\mathrm{G}$, Latte $\mathrm{A}$, Russo $\mathrm{M}$, et al. Determinazione fotometrica della cistinuria. Biochimica Clinica 2007; 31:267-71.

14. Latte A, Cangiano G, Russo M, et al. Determinazione del rapporto cistin/creatinina su AU400 Olympus. Biochimica Clinica 2007; 31:523.

15. Cangiano G, Latte A, Russo M, et al. Dosaggio al fosfotungstato di valori elevati di cistinuria. Biochimica Clinica 2009; 33:491.
16. Cangiano G, Di Maina E, Buccino G, et al. Ottimizzazione del dosaggio fotometrico della cistinuria. Biochimica Clinica 2013; 37:550.

17. Cangiano G, Paradisone C, Di Maina E, et al. Cistinuria al fosfotungstato. Nuovo dosaggio fotometrico a tre reagenti. Biochimica Clinica 2014; 38:460.

18. Marangella M, Petrarulo M, Daniele PG, et al. LithoRisk: software per il calcolo e la visualizzazione dei profili di rischio di calcolosi renale. G Ital Nefrol 2002; 6: 693-8.

19. Cangiano G, Latte A, Di Maina E, et al. Informatizzazione di laboratorio del rischio nefrolitiasico. Biochimica Clinica 2012; 36:602.

20. Cangiano G, Di Maina E, Buccino G, et al. Estrapolazione in Excel delle componenti del calcolo urinario. Biochimica Clinica 2013; 37:551. 